\title{
A type IVB secretion system adapted for bacterial killing, biofilm invasion and biocontrol
}

Gabriela Purtschert-Montenegro

Department of Plant and Microbial Biology, University of Zurich

\section{Gerardo Cárcamo-Oyarce}

Massachusetts Institute of Technology

Marta Pinto-Carbó

Department of Plant and Microbial Biology, University of Zurich

Kirsty Agnoli

Department of Plant and Microbial Biology, University of Zurich

Aurelien Bailly

Department of Plant and Microbial Biology, University of Zurich

Leo Eberl ( $\square$ leberl@botinst.uzh.ch)

Department of Plant and Microbial Biology, University of Zurich https://orcid.org/0000-0002-72410864

\section{Article}

Keywords: type IVB secretion system, Pseudomonas putida IsoF, bacterial killing, biofilm invasion, biocontrol

Posted Date: October 22nd, 2021

DOI: https://doi.org/10.21203/rs.3.rs-959170/v1

License: (c) (i) This work is licensed under a Creative Commons Attribution 4.0 International License. Read Full License

Version of Record: A version of this preprint was published at Nature Microbiology on September 19th, 2022. See the published version at https://doi.org/10.1038/s41564-022-01209-6. 


\section{A type IVB secretion system adapted for bacterial killing, biofilm invasion and}

\section{biocontrol}

Authors: Gabriela Purtschert-Montenegro ${ }^{1 \dagger}$, Gerardo Cárcamo-Oyarce ${ }^{1 \dagger \#}$, Marta PintoCarbó $^{1}$, Kirsty Agnoli ${ }^{1}$, Aurélien Bailly ${ }^{1}$ and Leo Eberl ${ }^{1 *}$

\section{Affiliations}

${ }^{1}$ Department of Plant and Microbial biology, University of Zurich, Zurich, Switzerland

†These authors contributed equally to this work

${ }^{\#}$ Current addresses: Department of Biological Engineering, Massachusetts Institute of Technology, Cambridge, Massachusetts, USA.

\section{*Correspondence to: leberl@botinst.uzh.ch}

\section{Abstract}

Many bacteria utilize contact-dependent killing machineries to eliminate rivals in their environmental niches. Here, we show that Pseudomonas putida IsoF is able to outcompete a wide range of bacteria with the aid of a novel type IVB secretion system (T4BSS) that can deliver toxic effectors into bacterial competitors. This extends the host range of T4BSSs, which were so far thought to transfer effectors only into eukaryotic cells, to prokaryotes. Bioinformatic and genetic analyses showed that this killing machine is entirely encoded by a rare genomic island, which has been recently acquired by horizontal gene transfer. IsoF utilizes this secretion system not only as a defensive weapon to antagonize bacterial competitors but also as an offensive weapon to invade existing biofilms, allowing the strain to persist in its natural environment. Furthermore, we show that IsoF can protect tomato plants against the plant pathogen Ralstonia solanacearum in a T4BSS-dependent manner, suggesting that IsoF capabilities can be exploited for pest control and sustainable agriculture. 
Many reports have demonstrated the great potential of microbial inoculants for bioremediation,

biofertilization and biocontrol applications ${ }^{1-4}$. However, the lab-to-field transition remains a

major limiting factor, since good in vitro performance is rarely reproduced in field trials. Microbial

survival, establishment and colonization are key features for biocontrol agents and applied microorganisms were often unable to persist in the environment or are rapidly outcompeted ${ }^{5-7}$.

Despite decades of research, the lack of understanding of intricate polymicrobial interactions has hampered the widespread use of inoculants in sustainable agriculture ${ }^{8-11}$. One important reason for the failure of a strain to colonize a desired niche is that the planktonic inoculants are unable to invade and persist in indigenous microbial consortia, which live within surfaceassociated communities, commonly referred to as biofilms ${ }^{5,12-15}$. Biofilm cells are embedded in an extracellular matrix that protects them from external stresses, like nutrient limitation, predation and the host immune response ${ }^{12,13,16,17}$. The extracellular matrix also restricts the entry of invaders into the biofilm interior and while bacteria can colonize and grow on the biofilm exterior, they are readily removed by shear forces ${ }^{18}$. Moreover, the ability to form biofilms is a widespread trait in plant-associated bacteria, that allows them to maintain a critical population in a specific location for periods sufficient to initiate beneficial or antagonistic interactions with the host plant ${ }^{19,20}$.

In addition, members of the indigenous biofilm consortium have evolved an arsenal of defense strategies $^{21}$ that limit the successful establishment of inoculants in the rhizosphere. Bacteria display two main strategies to antagonize invaders: the release of small molecules with antimicrobial activity into their surroundings ${ }^{22}$ and the delivery of toxic effector proteins through secretion systems into neighboring opponents, which relies on cell-to-cell contact ${ }^{23}$. Some of these effector molecules cause cell lysis by disrupting the cell envelope, while others are delivered into the cytoplasm where they affect DNA integrity or cell division, or exhaust energy resources ${ }^{24-30}$. To avoid self-intoxication by the effectors, bacteria produce cognate immunity

54 proteins, which are typically encoded by a gene localized in the vicinity of the effector gene $^{23,31,32}$. Various secretion systems are known to deliver effector proteins into bacterial competitors $^{23,31,33}$. The type $\mathrm{V}$ secretion systems (T5SS), encoded by the cdiBAl gene cluster, 
are widespread in Gram-negative bacteria. $\mathrm{CdiB}$ is an outer membrane $\beta$-barrel protein that exports the toxic CdiA protein, which forms a long filament extending from the cell surface. Upon binding of CdiA to specific receptors on the target cell, the C-terminal toxin domain is delivered into the bacterium to inhibit growth ${ }^{34,35}$. In Caulobacter crecentus, a type I secretion system (T1SS) exports the two bacteriocin-like proteins $\mathrm{CdzC}$ and $\mathrm{CdzD}$ to the surface of the producer cell where they form insoluble aggregates. These aggregates can drive contact-dependent killing of competitors by creating pores in the inner membrane 25,31 . The most versatile weapon, deployed by $25 \%$ of all Gram-negative bacteria, to target rival bacteria is the type VI secretion system (T6SS) ${ }^{36}$. It employs a contractile, phage-related nanomachine composed of several protein subcomplexes to inject diverse effectors into target cells in a one-step manner ${ }^{36-38}$. Most recently, a type IV secretion system (T4SS) was identified in Xanthomonas citri that is used to deliver an effector with peptidoglycan (PG) hydrolase activity, which lyses susceptible competitor cells ${ }^{26,31}$. There are two main classes of T4SS: i) The type IV A secretion systems (T4ASS), mostly used for DNA delivery and exemplified by the VirB/D4 system of Agrobacterium tumefaciens, and ii) the type IV B secretion systems (T4BSS), utilized to deliver effector proteins into their eukaryotic hosts and initially described as the Dot/lcm system found in intracellular pathogens such as Legionella pneumophila ${ }^{39-42}$. The two classes are only distantly related and T4BSS assemblies are larger than T4ASSs, comprising 27 components for the L. pneumophilla Dot/lcm system compared to 12 components for the VirB/D4 system ${ }^{42}$. In this study, we identified a T4BSS that can deliver toxic effectors into bacterial competitors, breaking the paradigm that T4BSSs are only used for effector transfer into eukaryotic cells ${ }^{43,44}$. This novel bacterial killing machine is encoded by a rare genomic island that was probably horizontally acquired by Pseudomonas putida IsoF, which is an effective colonizer of plant roots ${ }^{45,46}$. We demonstrate that IsoF utilizes this secretion system not only as a defensive weapon to antagonize phylogenetically diverse bacterial competitors but also as an offensive weapon to invade an existing biofilm by contact-dependent killing. We also show that IsoF can protect tomato plants against the pathogen $R$. solanacearum in a T4BSS-dependent manner, suggesting that this killing machine can be exploited for pest control. 
IsoF exhibits contact-dependent antagonism against a wide range of Gram-negative bacteria

We observed that $P$. putida IsoF (marked with Gfp; IsoF::Gfp) inhibited the growth of $P$. putida KT2442 (marked with mCherry; KT2442::mCherry) when culture samples were inoculated in a $1: 1$ ratio on a minimal medium plate. After 24 hours in contact-dependent competition (CDC), no red fluorescence could be observed from the macrocolony, indicating that the KT2442 had been outcompeted. We next determined the CFUs of the two strains after $24 \mathrm{~h}$ and $48 \mathrm{~h}$ and found that after two days IsoF had completely eliminated KT2442 (Fig. 1a). No adverse effect was seen when the two strains were separated by a $0.2 \mu \mathrm{m}$ pore size filter, suggesting that killing depends on cell-to-cell contact (Extended Data Fig. 1). To obtain further insight into the underlying molecular mechanism, we performed competition experiments on plates supplemented with propidium idiode $(\mathrm{PI})$, which allowed us to assess dead cells. After 48 hours of incubation, PI staining (magenta) was observed in the region where the two drops of the inoculated cultures overlapped, whereas dead cells were absent from the pure cultures regions (Fig. 1b). Time-lapse confocal laser scanning microscopy (CLSM) was used to demonstrate that KT2442 cells were killed after they had been in direct contact with IsoF::Gfp (Fig. 1c,

103 To determine the host range of the antagonistic activity of IsoF we fluorescently marked several 104 soil- and plant-associated bacteria as well as some phytopathogens, and tested whether they

105 are susceptible to killing by IsoF. All tested strains were outcompeted after $24 \mathrm{~h}$ of co-culture 106 with IsoF (Fig. 1d, e). Collectively, our data suggest that IsoF possesses a highly efficient, broad 107 host-range, contact-dependent killing machinery. IsoF utilizes a type IVB secretion system for bacterial killing

110 To identify the mechanism responsible for contact-dependent killing by IsoF, we constructed a 111 mini-Tn5 transposon insertion library of this strain and tested about 5,000 mutants for their 112 ability to outcompete $P$. aureofaciens::mCherry when grown as mixed macrocolonies (Fig. 2a). 
113 We identified 16 killing-defective insertion mutants (Extended Data Fig. 2). The transposon

114 insertion sites of eight of these mutants were determined by arbitrary $\mathrm{PCR}^{47}$ and were found to

115 be located in four genes of a large gene cluster, which we designated kib (killing, invasion, 116 biocontrol, see below), that appears to encode several elements of a T4BSS (Fig. 2b). While

117 T4BSSs of intracellular pathogens are well known for their capacity to deliver effectors to their

118 eukaryotic hosts ${ }^{42,48,49}$, they have so far not been reported to be involved in interbacterial killing.

119 To validate the results of the mutant screen, we constructed defined T4BSS mutants: $\triangle$ dotHGF,

120 which lacks the main structural components of the secretion system channel and $\Delta 23-\operatorname{trbN}$ 121 dotD, which lacks Piso_02323 encoding the hypothetical protein TrbN, which has a conserved 122 transglycosylase domain that has been proposed to assist DNA transfer across the PG in 123 conjugation systems $\mathrm{s}^{50}$. The latter mutant also lacked DotD, encoding a lipoprotein required for 124 assembly of the secretion system by positioning DotH in the outer membrane $e^{51-53}$. Single 125 mutants of Piso_02323 and dotD were also constructed and validated in CDC experiments. All 126 mutants tested had lost their ability to outcompete $P$. aureofaciens (Fig. 2d) and KT2442

127 (Extended Data Fig. 3), demonstrating that inactivation of the T4BSS apparatus prevents IsoF 128 from killing other bacteria.

The kib gene cluster is part of a unique genomic island present in only few Pseudomonas

\section{1 strains}

132 The region comprising the kib locus has a GC content of $58.8 \%$, whereas the IsoF genome has

133 an average GC content of $62.6 \%$ (Extended Data Table 1), which suggests the kib locus is part

134 of a genomic island that has been recently acquired by horizontal gene transfer. This hypothesis

135 was further strengthened by analyzing the IsoF genome with ICEfinder, allowing the detection

136 of mobile integrative and conjugative elements in bacteria ${ }^{54}$. This algorithm classified the IsoF 137 genomic island containing kib as a putative conjugative element, since it contains T4SS-related 138 genes and an integrase within the region. ICEfinder defined the borders of the gene cluster with 139 genes Piso_02313 and intA_3. Hence, the entire island has a size of 66,917 bp and encodes 14061 genes, 17 of which share homology with described T4BSS structural genes, 37 were defined 
141 as hypothetical proteins, and four encode a Type I Restriction Modification (RM) system and an

142 integrase at the 3'-end of the island (Fig. 2b,c). The basic local alignment search tool (BLAST)

143 was used to interrogate the cluster and revealed that kib genes are also present in 11 other

144 Pseudomonas strains, 10 of which are environmental isolates and one is a clinical isolate. Eight

145 of these strains were classified as P. putida (Extended Data Table 1). Interestingly, all

146 orthologous kib gene clusters showed conserved synteny and were located at the same

147 chromosomal position (Extended Data Fig. 4), suggesting a common ancestor. Notably, the

148 orthologous clusters showed deletions at the 3'-end of the island, including the Type I RM

149 system and the flanking integrase (Extended Data Figs. 4 and 5). Previous work has shown

150 that the genes encoding components of T4BSSs are organized in smaller clusters that are

151 distributed across the chromosome or plasmid of various bacteria ${ }^{49}$. In contrast, the kib locus

152 appears to encode all components of the T4BSS and we were unable to identify additional

153 genes potentially encoding other components of the secretion system in the genome of IsoF

154 (Extended Data Fig. 5). These data reinforce the idea that the kib gene cluster has only recently

155 been acquired via horizontal gene transfer and encodes all components of the bacterial killing

156 machine.

158 The $k i b$ gene cluster encodes an effector-immunity (E-I) pair

159 Contact-dependent killing systems deliver toxic effector molecules into bacterial competitors.

160 To avoid self-killing, the attacking bacterium produces a cognate immunity protein that 161 neutralizes the toxin. The immunity and toxin genes form a so-called effector-immunity (E-I)

162 pair, and are often co-transcribed ${ }^{22,32,55,56}$. The kib gene cluster does not contain any previously

163 described or obvious candidate toxin genes but many hypothetical genes (Fig. 2b), which could

164 potentially encode effector molecules. However, as effector proteins are very heterogeneous

165 both in sequence and function ${ }^{22,57}$, we were not able to identify promising candidates using in

166 silico analysis. To investigate whether an E-I pair was present within the kib region, we deleted

$16749.5 \mathrm{~kb}$ of the genomic island containing all kib genes (Piso_02313 to Piso_02360, Extended

168 Data Fig. 5). The resulting mutant, designated $\Delta \mathrm{T} 4 \mathrm{~B}$, no longer killed $P$. aureofaciens and 
169 formed mixed macrocolonies with this strain (Fig. 2d). Interestingly, the $\Delta T 4 B$ mutant became

170 susceptible to KT2442 in co-culture on nutrient plates, likely because KT2442 possesses a

171 T6SS that was shown to efficiently kill other bacteria ${ }^{27}$ (Extended Data Fig. 3). Our original

172 observation that IsoF kills KT2442 (Fig. 1a), suggests that kib-mediated killing is faster or more

173 efficient than T6SS-mediated killing of KT2442. We hypothesized that the absence of an E-I

174 pair would render the $\triangle \mathrm{T} 4 \mathrm{~B}$ mutant sensitive to the wild type strain, while its presence would

175 confer resistance. In competition experiments between IsoF and $\Delta T 4 \mathrm{~B}$, the mutant strain was

176 indeed killed, while it was able to co-exist with the $\triangle$ dotHGF mutant, which lacks the structural

177 components of the secretion channel required for killing (Fig. 3a). These experiments

178 demonstrate that the genes required for killing and self-protection are present within the kib

179 cluster. Moreover, IsoF was unable to kill mutants $\Delta$ dotHGF and $\Delta 23-\operatorname{trb} N$-dotD, indicating that

180 both deletion mutants are protected against effector toxicity from the wild type strain and that

181 no immunity gene was located within the regions deleted in these mutants (Extended Data Fig.

$1826)$.

183

184 Identification of a kib immunity protein through transposon sequencing

185 Immunity genes are essential since cells lacking an immunity protein would either be killed by 186 neighboring bacteria or die due to self-intoxication ${ }^{32,58}$. We therefore reasoned that it should be

187 possible to identify the genetic elements required for self-protection by transposon sequencing.

188 This approach has previously been employed to identify E-I pairs of T6SSs in V. cholerae and

$189 P$. aeruginosa ${ }^{58,59}$. To this end, we generated a saturated transposon insertion library in the IsoF

190 wild type strain. The pooled library, which consisted of approximately 700,000 mutants, was

191 subjected to three different growth regimes: (i) growth in liquid medium with shaking to prevent

192 cell-to-cell contact, (ii) growth on an agar surface either alone or (iii) in the presence of the

193 competitor P. aureofaciens to promote competition (Fig. 3b). Sequencing of the genomic DNA

194 resulted in more than 7 million reads per sample as summarized in Extended Data Table 2. We

195 used the unique insertion density approach of the Tn-Seq explorer software to identify genes

196 that provide a fitness benefit for growth under the different growth regimes ${ }^{60}$. This analysis 
197 identified one gene, Piso_02332, within the kib region, which was virtually devoid of transposon

198 insertions in all three treatments (Fig. 3c, Extended Data Table 3). This gene appears to be co-

199 transcribed with Piso_02333, possibly constituting a novel E-I pair.

200 To determine the role of this putative E-I pair in bacterial killing, we deleted both genes

201 in IsoF to generate $\Delta 32-33$. We were also able to delete the putative effector gene, giving rise

202 to mutant $\Delta 33$. Unexpectedly, we noticed that the $\Delta 32-33$ grew slower on $A B C$ minimal media

203 relative to the parental strain or mutant $\Delta 33$ (Extended Data Fig. 8). In order to establish a fair

204 competition situation despite the growth difference, the CDC assays with $\Delta 32-33$ were 205 performed on ABC medium supplemented with casamino acids and the CFUs were normalized 206 to the number of cells recovered from the monoculture of $\Delta 32-33$ after $24 \mathrm{~h}$. Importantly, $\Delta 33$ 207 was unable to compete with $P$. aureofaciens or KT2442 and complementation partially rescued 208 the killing phenotype, suggesting that Piso_02333 encodes a toxic effector protein. This is 209 further supported by the lack of growth inhibition of $P$. aureofaciens and KT2442 by the double 210 mutant $\Delta 32-33$, although we were unable to restore killing by complementation (Extended Data 211 Fig. 9a, b). In competition against IsoF wild type, mutant $\Delta 33$ and its complemented derivative 212 survived (Fig. 3d), indicating that both strains are immune to the IsoF effector toxin. By contrast, 213 mutant $\Delta 32-33$ was outcompeted by IsoF, while the complemented strain co-existed with IsoF 214 (Fig. 3d), indicating that Piso_02332 confers immunity to kib-mediated killing. To further test 215 this possibility, mutants $\Delta \mathrm{T} 4 \mathrm{~B}$ and $\Delta 32-33$ were complemented with Piso_02332 on a plasmid 216 (pBBR::32) and the resulting strains were used in competition assays against IsoF. While strain $217 \Delta 32-33 / p B B R:: 32$ co-existed with IsoF, mutant $\triangle T 4 B / p B B R:: 32$ was outcompeted (Fig. 3e). 218 This suggests that while Piso_02332 confers resistance to Piso_02333, kib may encode an 219 additional effector that is not neutralized by Piso_2332.

\section{The kib killing system enables IsoF to invade an established biofilm}

222 We hypothesized that contact-dependent competition might be an efficient way to eliminate 223 competitors in communities such as polymicrobial biofilms. Since both IsoF and KT2442 are 224 good biofilm producers, they represent a good proxy for evaluating the role of kib-mediated 
competition in mixed-species biofilms. To investigate this, we first established a KT2442::Gfp

226 (green) biofilm in a flow cell system and then introduced IsoF::mCherry (blue) (Fig. 4a). Within 227 one day, IsoF cells attached to the surface began to proliferate and formed numerous 228 microcolonies. After 3 days of incubation IsoF had formed a mature biofilm by invading and displacing the KT2442 biofilm. The volume of KT2442 biofilm decreased by approximately 40

$230 \%$ between 72 and $96 \mathrm{~h}$ post IsoF inoculation, which reached the equal biomass with KT2442 231 after two days of competition (Fig. 4b). Without competition, the biomass of the KT2442 biofilm 232 increased steadily over time (Extended Data Fig. 10a, b). When a pre-established KT2442 233 biofilm was challenged with the kib mutants $\triangle$ dotHGF or $\Delta 23-t r b N-d o t D$, neither of the mutants 234 was able to form microcolonies or to invade the existing biofilm (Fig. 4a, b). Importantly, $235 \Delta$ dotHGF or $\Delta 23-t r b N-d o t D$ mutants in isolation formed biofilms similar to the IsoF wild-type 236 strain (Extended Data Fig. 11a, b). We hypothesized that IsoF employed its T4BSS to kill 237 KT2442 cells upon contact within the biofilm, creating space for the expansion of the IsoF 238 biofilm. To test this, we inoculated flow cells with IsoF::Cfp (cyan) and KT2442::Gfp (yellow) 239 with equivalent numbers of cells and monitored the fate of KT2442 microcolonies neighboring 240 IsoF microcolonies by adding PI (red) as an indicator of cell death. As shown in Figure 4e, dead 241 cells were observed at positions where the two strains were in direct contact. We determined 242 that the biofilm volume of KT2442 was reduced by approximately $20 \%$ (Fig. 4f). We next 243 visualized killing of KT2442 by IsoF::Gfp in a mixed monolayer biofilm on the surface of a 244 minimal medium agar pad (Fig. 4c). After $18 \mathrm{~h}$ incubation we observed that nearly $92 \%$ of the 245 dead KT2442 cells present (magenta) were located next to IsoF::Gfp (green) cells, as opposed 246 to those that were not in contact with a green cell, demonstrating that kib-mediated killing is 247 strictly dependent on cell-to-cell contact (Fig. 4d). By contrast, when KT2442 was challenged 248 with either the $\Delta$ dotHGF or the $\Delta 23-\operatorname{trbN}$-dotD mutant (green), very few dead cells were 249 observed, similar to monoculture biofilm controls (Fig. 4d, Extended Data Fig. 12). In 250 conclusion, these results suggest that the kib system not only allows IsoF to defend itself against 251 competitors but also to kill bacteria that live within an established biofilm community, which 252 eventually becomes replaced by the biofilm of the invading IsoF strain. 
254 Kib-mediated killing allows IsoF to protect tomato plants from the phytopathogen Ralstonia solanacearum

256 Since IsoF was initially isolated from the rhizosphere of tomato plants and was shown to efficiently colonize the root surface ${ }^{46}$, we assessed whether the kib killing system could be useful for the biocontrol of $R$. solanacearum, a major pathogen causing bacterial wilt in tomato, amongst a wide range of other crops $^{61,62}$. In vitro competition experiments showed that IsoF outcompeted $R$. solanacearum, while the $\Delta$ T4B mutant did not (Fig. 1d, e, Extended Data Fig. 13). We next tested whether IsoF could also protect tomato plants from $R$. solanacearum infection. Considering that $R$. solanacearum is a soil-borne pathogen which enters the plant through natural openings like emerging lateral roots or wounds ${ }^{61}$, we injured established tomato seedlings with small incisions (Fig. 5a). Twenty-two days post infection, control plants inoculated with $R$. solanacearum were severely wilted, with signs of chlorosis and arrested development of the root and shoot systems. By contrast, $90 \%$ of the seedlings inoculated with a mixture of $R$. solanacearum and IsoF showed no signs of wilting (Fig. 5b). However, when seedlings were co-inoculated with a mixture of $R$. solanacearum and the kib mutant $\Delta \mathrm{T} 4 \mathrm{~B}$, wilting and underdevelopment were observed in $85 \%$ of the plants, indicating that IsoF prevented $R$. solanacearum from spreading into the plant tissues by kib-mediated killing. To 271 precisely evaluate wilt development, we determined the chlorophyll content and measured 272 shoot area and root weight of individuals from the treatment groups as a proxy for plant health. 273 These data were subjected to principal component analysis and hierarchical clustering (Fig. 5b, 274 c). The two first components accounted for $94.6 \%$ of the variance and a score scatter plot 275 clearly clustered the single inoculations with IsoF and $\triangle T 4 \mathrm{~B}$ groups together with untreated plants, confirming that the strains do not harm tomato plantlets. Plants co-inoculated with $R$.

277 solanacearum and IsoF preferentially clustered with healthy plants while those co-inoculated 278 with $\Delta$ T4B grouped with $R$. solanacearum infected plants. This clearly indicates that IsoF 279 decreases the pathogen load in the injured tomato tissues in a kib-dependent manner. To verify 280 that IsoF indeed killed $R$. solanacearum, we recovered the bacteria attached to the roots and 
determined the CFUs. This showed that the number of $R$. solanacearum cells present after co-

282 inoculation with IsoF was significantly lower than after inoculation with the $\Delta T 4 B$ mutant (Fig.

$2835 \mathrm{~d}$ ). Together, these results demonstrate that the biocontrol capacity of IsoF against bacterial 284 pathogens such as $R$. solanacearum depends on the kib locus.

\section{Discussion}

In this study, we show that $P$. putida IsoF uses a T4BSS to kill a wide range of soil and plantassociated Gram-negative bacteria in a contact-dependent manner. This killing machinery enables IsoF to invade and replace pre-established biofilms and to protect tomato plants from the phytopathogen $R$. solanacearum. Consequently, we have named the gene cluster encoding this T4BSS kib (killing, invasion and biocontrol). While previous work has demonstrated that the opportunistic pathogen Stenotrophomonas maltophilia, the plant pathogen Xanthomonas citri and the animal pathogen Bartonella schoenbuchensis possess T4ASSs that kill other bacteria $^{26,63,64}$, this is the first report of a T4BSS that is used for interbacterial killing. T4BSSs are employed by various pathogens to translocate effector molecules into their eukaryotic host cells $\mathrm{s}^{42,48,49}$ and thus kib extends the host range of T4BSSs to prokaryotes.

Our bioinformatic and molecular analyses showed that the kib locus encodes all components of the killing machinery. This contrasts with other killing systems, where the genetic components are often spread across the chromosome. For example, the loci encoding the structural genes of the Xanthomonas and Stenotrophomonas T4ASSs are separated from the genes encoding the effectors ${ }^{63,65}$. A similar separation has been reported for the Legionella T4BSS, which is encoded in numerous clusters distributed over the entire genome and the plasmids ${ }^{49,66}$. Our data suggest that kib is part of a genomic island and the striking difference in GC content between the island and the IsoF core genome (4.6\%), together with the presence of genes encoding an integrase as well as a Type I RM system flanking kib, suggest that it was recently

307 acquired by horizontal gene transfer (Fig. 2b, Extended Data Table 1). This is also in line with 308 the finding that orthologs of kib were only present in 11 Pseudomonas strains. Interestingly, all 
of these homologous islands lacked the Type I RM system and the integrase (Extended Data Fig. 5).

312 We speculated that the kib cluster provides IsoF a competitive advantage for survival in the environment, as IsoF was shown to be an excellent colonizer of tomato roots and most of the

314 other strains carrying kib were also isolated from soil. Our results showed that IsoF indeed 315 outcompeted various environmental strains, most notably P. putida KT2442, which was recently 316 demonstrated to use its K1-T6SS as an antibacterial killing device ${ }^{27}$. IsoF does not harbor a 317 homolog of the K1-T6SS gene cluster and thus was expected to be sensitive to killing by $318 \mathrm{KT} 2442$. In fact, the $\triangle \mathrm{T} 4 \mathrm{~B}$ mutant of IsoF was found to be killed by KT2442 in co-culture. 319 However, when the two wild type strains were competed against each other, IsoF eliminated 320 KT2442, indicating that T4BSS-mediated killing may occur before KT2442 can fire its T6SS 321 apparatus (Fig. 1a, Extended Data Fig. 3). Previous work has shown that bacteria have different 322 strategies for deploying their T6SS. While some strains of Vibrio cholerae use their T6SS in an 323 untargeted fashion and assemble and fire their apparatus in random locations within the cell, $P$. 324 aeruginosa assembles and fires its organelle only after detecting an attack from another nearby 325 bacterium $^{67,68}$, a strategy that has been termed the T6SS tit-for-tat response ${ }^{69}$. More recent work 326 has provided evidence that $P$. aeruginosa senses outer membrane perturbations caused by the 327 attack of competitors, treatment with the membrane-targeting antibiotic polymyxin, or 328 interference with outer membrane biogenesis via a signal transduction pathway that triggers 329 the tit-for-tat response ${ }^{70}$. At present, neither the triggers of the KT2442 K1-T6SS nor of the IsoF 330 kib system are known. However, that IsoF kills KT2442 may indicate that kib is constitutively 331 expressed and fires in a random fashion, while the K1-T6SS of KT2442 is only activated upon 332 attack. This would be reminiscent of the finding that a T6SS-negative $V$. cholerae strain is not 333 killed by $P$. aeruginosa whereas $V$. cholerae is efficiently killed in co-cultures with $P$. aeruginosa 334 when both organisms contain a functional $\mathrm{T} \mathrm{SS}^{69}$. While this would explain why all defined kib 335 mutants of IsoF co-existed with KT2442, it does not explain why the $\Delta$ T4B mutant, which lacks 336 the entire kib locus, was killed by KT2442. Additional work will be required to elucidate whether 
differences in the triggers or efficacies of the killing systems are responsible for the superior performance of the IsoF kib system. In this context, it is worth noting that IsoF killed many bacteria that were shown to use T6SSs for interbacterial competition, including $P$. aeruginosa

B. cenocepacia $\mathrm{H} 111^{71}$, P. syringae ${ }^{72}, P$ P. chlororaphis ${ }^{73}$ P. fluorescens ${ }^{74}, P$. carotovorum $^{75}$, E. amilovora ${ }^{76}$ and B. thailandensis ${ }^{77}$ (Extended Data Fig. 14).

We performed a Tn-Seq analysis to identify potential E-I pairs within the kib gene cluster. This 344 strategy, which assumes that inactivation of an immunity gene is lethal for the cell, identified Piso_02332 as an essential gene. Importantly, we found this gene to be essential for growth under all conditions tested, namely in liquid medium, in a macrocolony on the surface of an agar plate or in the presence of a competitor strain, suggesting that this gene is constitutively expressed. We provided evidence that Piso_02332 encodes an immunity protein that neutralizes the toxicity of an effector protein encoded by Piso_02333, which appears to be cotranscribed with Piso_02332, a genetic architecture that is frequently found with E-I pairs $22,32,55,56$. A defined Piso_02333 mutant killed neither KT2442 nor P. aureofaciens and this defect was at least partially restored by genetic complementation (Extended Data Fig. 9). At present the cellular target of the Piso_02333 effector is unknown. Given that cells killed by IsoF maintained their shape and did not lyse, it is tempting to speculate that the target is located in 355 the cytosol rather than the cell envelope. Expression of Piso_02332 in the $\Delta 32-33$ mutant 356 conferred immunity against the IsoF wild type strain, indicating that Piso_02332 neutralizes the 357 toxicity of the Piso_02333 effector. Unexpectedly, complementation of $\Delta T 4 \mathrm{~B}$, which lacks the 358 entire kib locus, did not protect the mutant against IsoF, suggesting that an additional E-I pair may be encoded by the kib gene cluster (Fig 3e). We noticed that the transposon insertion density of another gene within the kib locus, Piso_02351, was reduced, albeit to a lesser degree 361 than Piso_02332 (Extended Data Fig. 7, Extended Data Table 3). Whether this gene together 362 with one of its adjacent genes could comprise another E-I pair remains to be elucidated.

364 Natural biofilms have dynamic and heterogeneous structures that are shaped by both 
environmental forces and microbial interactions, which may be cooperative or antagonistic ${ }^{78-80}$.

366 The biofilm matrix protects the cells from various external stresses and restricts the entry of 367 invaders into the biofilm ${ }^{18,78}$. Moreover, many bacteria use defense mechanisms that would 368 effectively kill competitors that attempt to enter the established biofilm community ${ }^{22,23,33}$. In this study we demonstrate that $P$. putida IsoF has the unprecedented ability to invade and replace an established biofilm of $P$. putida KT2442. However, when the two wild type strains competed

371 against each other, KT2442 was eliminated, presumably because it was killed before it could 372 fire its T6SS. In accordance with a recent report ${ }^{81}$, we observed that on agar plates dead cells 373 created a barrier that prevented further killing. This was not observed when the biofilms were 374 grown in flow cells, as dead KT2442 cells detached from the glass substratum and were removed by the shear forces of the nutrient flow. The freed space was then occupied by IsoF, 376 which eventually led to the replacement of the existing KT2442 biofilm (Fig. 4a, b). It is worth 377 mentioning that IsoF produces the powerful biosurfactant putisolvin, which was previously 378 shown to promote surface translocation and biofilm expansion ${ }^{82}$. Whether putisolvin is involved 379 in the removal of dead KT2442 cells or the movement of IsoF cells into the freed spaces in the 380 KT2442 biofilm is another interesting topic for future investigations.

382 IsoF was originally isolated from the rhizosphere of a tomato plant and was shown to form a 383 biofilm on root surfaces ${ }^{83}$. Here, we demonstrate that IsoF is able to antagonize several 384 economically relevant phytopathogens (Fig. 1d, e). Moreover, we show that IsoF can protect 385 tomato plants from the soil-borne pathogen $R$. solanacearum, which can infect over 250 386 different plant species, among them important agricultural crops $^{61}$ (Fig 5). The presence of 387 microbes secreting bacteriocins, antifungals or antibiotics in the rhizosphere was shown to be 388 an effective strategy to suppress plant pathogens ${ }^{21,80,84}$. In this study we demonstrate that IsoF 389 uses its T4BSS not only to kill phytopathogens but also to invade biofilms. Given that a major 390 limitation in biocontrol applications is that inoculants are unable to establish themselves in the 391 environment, IsoF, which utilizes kib for attack as well as for defense, is a very strong candidate 392 for a novel bioinoculant for plant protection. 


\section{Methods}

\section{Bacterial growth conditions and media}

396 Bacterial strains used in this study have been listed in Table 1. Most bacterial overnight 397 cultures were grown in Lysogeny Broth (LB, Difco) at $30^{\circ} \mathrm{C}$ (Pseudomonas species) or at $37^{\circ} \mathrm{C}$ 398 (Escherichia coli). Ralstonia solanacearum, Pectobacterium carotovorum, Pseudomonas 399 syringae overnight cultures and experiments were done in LB media without salt (LB-, Difco) 400 at $30^{\circ} \mathrm{C}$. All other experiments were performed in $A B$ medium ${ }^{85}$ supplemented with $10 \mathrm{mM}$ 401 sodium citrate (indicated as ABC medium). If indicated, ABC was supplemented with $4 \mu \mathrm{g} \mathrm{ml}$ 4021 propidium iodide (PI, Thermo Fisher). Additionally, ABC was supplemented with $0.2 \%$ 403 casamino acids if indicated (ABCAS). For selection of Pseudomonas mutants or 404 transconjugants, Pseudomonas Isolation Agar (PIA, Difco) was used. If required, antibiotics 405 were added at the following final concentrations: for E. coli: $100 \mu \mathrm{g} \mathrm{ml}^{-1}$ ampicillin (Amp), 25 $406 \mu \mathrm{g} \mathrm{ml} l^{-1}$ kanamycin (Km), $10 \mu \mathrm{g} \mathrm{ml}^{-1}$ gentamycin (Gm), $10 \mu \mathrm{g} \mathrm{ml}^{-1}$ tetracycline (Tc); for 407 Pseudomonas species: 75 or $100 \mu \mathrm{g} \mathrm{ml}^{-1}$ kanamycin, 20 or $30 \mu \mathrm{g} \mathrm{ml}^{-1}$ gentamycin, $20 \mu \mathrm{g} \mathrm{ml}{ }^{-1}$ 408 tetracycline.

\section{Construction of fluorescently tagged strains}

411 The mini-Tn7 system ${ }^{86}$ was employed to integrate the gene encoding red fluorescent protein 412 (mCherry) or green fluorescent protein (Gfp), into the chromosome of the strains listed in Table

413 1. Mini-Tn7 tagged strains were obtained by tri-parental mating using the donor strain E. coli 414 S17-1 carrying pUCT18-mini-Tn7 and the helper plasmid pUX-BF13 ${ }^{87}$. Briefly, overnight 415 cultures of the recipient strain, the helper strain and the donor strain were washed with $0.9 \%$ $\mathrm{NaCl}$ and then mixed in a 1:2:2 ratio (recipient : helper : donor). The strains were inoculated on LB plates as $50 \mu \mathrm{l}$ drops and incubated at $30^{\circ} \mathrm{C}$ overnight. Bacteria were resuspended in 1 $\mathrm{ml} 0.9 \% \mathrm{NaCl}$ and plated on media containing $\mathrm{Gm}$. Plates were incubated overnight at $30^{\circ} \mathrm{C}$ and fluorescent colonies were selected. 


\section{Tn5 mutant library, screening and mutant identification}

422 A transposon mutagenesis was performed as previously described ${ }^{88,89}$, using IsoF as the 423 genetic background and the transposon delivery vector pUT/mini-Tn5 $\mathrm{Km}^{90}$. Approximately 42440,000 independent transposon insertion mutants were obtained. Aliquots of the library were saved and stored at $-80^{\circ} \mathrm{C}$. To perform the screening, individual mutants were grown overnight in $100 \mu \mathrm{LB}$ on 96-well plates, then the cultures were gently combined with $100 \mu \mathrm{l}$ of $P$.

427 aureofaciens::mCherry. The mixed- inocula were transferred to ABC medium agar plates using 428 a 96-pin replicator. Approximately 5,000 single Tn5 mutants were independently co-inoculated with $P$. aureofaciens::mCherry and incubated for $24 \mathrm{~h}$ at $30^{\circ} \mathrm{C}$. Mixed bacterial colonies were examined by means of fluorescence microscopy where competitions that showed red fluorescence indicated Tn5 mutants defective in killing. Initial hits were validated by contactdependent competition assays as described later. Identification of the Tn5 insertion mutants was done by arbitrary PCR as described by Espinosa-Urgel et al, $2000^{47}$. After the second

434 round of PCR, reactions were cleaned with the PCR Purification Kit (Qiagen) and sequenced. 435 Sequences were analysed and compared with the genome of IsoF and with NCBI Blast. The whole genome sequence of IsoF has been uploaded and is publicly available on NCBI under the accession number CP072013.

\section{Contact-dependent competition (CDC) assays}

440 Overnight cultures were adjusted to an $\mathrm{OD}_{600}$ of 1 and dilutions were made to determine the 441 number of colony forming units (CFUs) of each competitor. For the CDC assays, competitors 442 were mixed in a 1:1 CFU ratio. ABC, ABCAS, or LB medium was inoculated with $5 \mu$ of mixed 443 culture. To determine the bacterial population in the mixed macrocolonies, CFU were counted 444 at $0 \mathrm{~h}$ and at $24 \mathrm{~h}$. At $24 \mathrm{~h}$, two macrocolonies were resuspended in $500 \mu \mathrm{l} \mathrm{of} 0.9 \% \mathrm{NaCl}$ and serial dilutions were plated on PIA and PIA Gm, the latter to select tagged strains. For the macrocolony overlaying competition, IsoF::Gfp was inoculated first and incubated at $30^{\circ} \mathrm{C}$ for

$4471 \mathrm{~h}$, then KT2442 was inoculated to cover half of the IsoF colony. Fluorescence of the mono and mixed cultures was examined using a Leica M165 FC Fluorescence Stereo Microscope. 


\section{$450 \quad$ Single cell competitions}

451 On a microscope slide, $8-9 \mathrm{~mm} \varnothing \times 1 \mathrm{~mm}$ depth adhesive silicon isolators (Grace BioLabs) 452 were attached and filled with $62 \mu \mathrm{ABC}$ with $0.7 \%$ agar supplemented with PI. The middle of 453 the agar was inoculated with $1 \mu$ of the bacterial 1:1 mixed culture (IsoF::Gfp : competitor 454 strain). The cover slip was placed on top after the inoculant had dried, and competition was 455 monitored with a confocal laser scanning microscope every $15 \mathrm{~min}$ for about $3 \mathrm{~h}$. A final time 456 point was recorded at after 18-22 hours of incubation at RT including samples of the mono 457 cultures. Image acquisition was done using a confocal laser scanning microscope (CLSM, 458 Leica TCS SPE, DM5500) equipped with a x100/1.44 oil objective. Images were analysed with 459 Image ${ }^{91}$.

\section{Construction of $\boldsymbol{P}$. putida IsoF deletion mutants}

462 IsoF derivatives with single and triple gene deletions and the deletion of the kib cluster (49.5 $463 \mathrm{kbp}$ ) were constructed using Sce-l based mutagenesis as described in Flannagan et al, $2008^{92}$. 464 First, the plasmid pGPI-Scel (which carries an I-Scel recognition site) was modified by cloning 465 tetAR, which encodes a tetracycline efflux pump into the Pstl restriction site to give pGPI466 Scel::TetAR. Next, two homology regions flanking the region to be deleted were cloned into pGPI-Scel::TetAR. The plasmid was introduced via conjugation and integrated into the genome of $P$. putida IsoF by single homologous recombination, giving two copies of the homologous regions in the chromosome. The plasmid pDAl::Gm ${ }^{R}$, which carries the I-Scel 470 nuclease, was then conjugated into the single-crossover IsoF strain. The I-Scel nuclease 471 produced a double strand DNA break at its recognition site, linearising the chromosome and 472 requiring recombination for the survival of the cell. This occurred preferentially at the repeated 473 homologous regions. For both conjugations, the pRK2013 helper plasmid was used to provide 474 the genes encoding the conjugation machinery. Ex-conjugants were selected on PIA Gm 
476 Gm20 to select colonies from which the pDAl plasmid had been cured. All primers and 477 restrictions enzymes used for cloning are listed in Supplementary Table 2.

\section{Construction of pBBR1MCS derivative plasmids}

480 For complementation of the $\Delta 32-33$ and $\Delta 33$ mutants, plasmids pBBR::32-33 and pBBR::33 481 were constructed. Additionally, pBBR::32 was constructed. In each case the coding sequence 482 plus the native promoter region was amplified using an IsoF cell lysate as a template and 483 cloned into pBBR1MCS-2 using primers and restriction sites as listed in Supplementary Table 4841 and 2. E. coli MC1061 was transformed with the ligated vectors, which were then transferred 485 into the IsoF deletion mutants by triparental mating using E. coli DH5a pRK2013 as the helper strain. Complementation was checked by colony PCR using primers listed in Supplementary

487 Table 2.

\section{Comparative genomic analysis}

Identification of Pseudomonas strains carrying the T4BSS gene cluster elements was performed using NCBI BLAST. The online tool ICEfinder was used to determine the boundaries of IsoF's genomic island (GI). The region containing the IsoF GI was compared against the 11 other Pseudomonas strains carrying T4BSS elements using the MAUVE alignment tool with

494 the default settings ${ }^{93}$. MultiGeneBlast was done using the 11 Pseudomonas strains, IsoF, and 4958 additional known species in which the T4BSS has been described ${ }^{94}$. Alignment and 496 comparison were done using the following settings: Gene identity threshold $30 \%$, number of 497 hits mapped 1000 and maximum distance between the genes in a locus $10 \mathrm{~kb}$ to search for 498 tightly coupled operons.

\section{$500 \quad$ Tn-Seq methodology}

501 Transposon mutagenesis was performed by tri-parental conjugation. First, the resistance 502 properties of the donor plasmid pLG99 (carrying a Tn23 transposon) were modified by cloning 503 a kanamycin resistance gene into the Aatll restriction site. Overnight cultures of the recipient 
504 strain P. putida IsoF, the helper strain E. coli DH5a pRK2013 and the donor strain $E$. 505 coli CC118 $\lambda$-pir pLG99::Km were washed with $0.9 \% \mathrm{NaCl}$ and then mixed in a 1:2:2 ratio 506 (recipient : helper : donor). The conjugation was plated on LB plates in drops of $50 \mu$ and 507 incubated for $2 \mathrm{~h}$ at $37^{\circ} \mathrm{C}$, followed by incubation at $30^{\circ} \mathrm{C}$ overnight. The mating drops were 508 resuspended in $6 \mathrm{ml} 0.9 \% \mathrm{NaCl}$ and plated on PIA containing $\mathrm{Km}$. Plates were incubated at $50930^{\circ} \mathrm{C}$ overnight and the resulting colonies were washed from the plate with LB supplemented with $\mathrm{Km}$. The resuspended mutant library was then mixed with an equal amount of $50 \%$ 511 glycerol and kept at $-80^{\circ} \mathrm{C}$. From three independent conjugations with approximately 70 512 matings, an estimated 700,000 mutants were generated.

For the Tn-Seq experiments, the pooled mutant library was first grown for $16 \mathrm{~h}$ in liquid $A B C$ media supplemented with $0.2 \%$ rhamnose until stationary phase. The $O D_{600}$ was then adjusted to 0.05 for growth in liquid medium (condition 1 ) and to an $\mathrm{OD}_{600}$ of 1 for growth on solid medium as a monoculture (condition 2) and on solid medium as a co-culture (condition 3). For the growth in liquid medium the Tn library was incubated at $30^{\circ} \mathrm{C}$ for $4.5 \mathrm{~h}$ with $220 \mathrm{rpm}$ shaking, then cells were collected and pelleted for DNA extraction. For treatments 2 and 3 , 400 drops of $5 \mu \mathrm{l}$ each of the bacterial culture were plated. For the mixed condition (condition 3), $P$. aureofaciens was co-inoculated with the Tn mutant library in a 1:1 CFU ratio. Both plated conditions were incubated for $8.5 \mathrm{~h}$ at $30^{\circ} \mathrm{C}$. Cells were scraped from the plate with $0.9 \% \mathrm{NaCl}$ and adjusted to an $\mathrm{OD}_{600}$ of 2 before being pelleted and kept at $-20^{\circ} \mathrm{C}$ for later $\mathrm{DNA}$ extraction. DNA extraction was done using the Bacterial Genomic DNA Kit (Sigma-Aldrich). All sequencing steps were performed using the circle method described by Gallager et al, $2011^{95}$ with several modifications described in Higgins et al, $2020^{96}$.

\section{Tn-Seq data analysis and bioinformatics}

528 The Illumina sequencing reads were trimmed using Trimmomatic-0.32 (Leading: 30, Trailing: 529 30, Slidingwindow: 4:20, Minlen: 60 ${ }^{97}$. Adapter sequences were removed with Cutadapt $530 \mathrm{v1.9^{98 }}$. Tn-Seq Explorer was used to analyse the resulting Tn-Seq data ${ }^{60}$. NCBI protein (.ptt) and RNA (.rnt) table files were generated from the IsoF genbank file (.gbff) and provided as 
532 input to Tn-Seq Explorer in order to infer the coordinates of proteins and RNA coding regions.

533 Trimmed reads were mapped to the chromosome using the Bowtie 2 plugin of Tn-Seq explorer

534 (--very-sensitive- command) ${ }^{99}$. A sequence alignment map (SAM) file was produced. For each dataset, the subsequent sequence alignment map (SAM) generated with Bowtie2 was evaluated by $\mathrm{Tn}$-Seq Explorer to assess essentiality. In the analysis, transposons mapping within $5 \%$ of the start codon and $20 \%$ of the stop codon were excluded. An estimated cut-off

538 UID (unique insertion density) was established in order to separate essential from nonessential genes ${ }^{60}$. This was done by dividing the number of unique insertions by the gene

540 length, resulting in the UID for that specific gene. The number of genes with the given insertion

541 density versus the insertion density per bp was represented in a plot, usually showing a 542 bimodal distribution. Here the genes with low or no-insertions appeared on the left side of the

543 plot. The point where the plot rises again indicates the threshold for genes that can tolerate

544 transposon insertions ${ }^{95}$. This point indicates the cut-off for essential genes, which was set for 545 each growth condition: Liquid $=0.013$, Plate $=0.011$ and Mixed $=0.014$ (UID). Genes showing 546 higher UID values were considered non-essential since high number of transposon insertions 547 were detected per gene.

\section{Availability of sequencing data}

550 FASTQ files generated from the Illumina MiSeq platform are publicly available at the NCBI 551 short reads archive (SRA) under the BioProject: PRJNA730700. Individual datasets have 552 following accession numbers: Liquid: SRR14612110, Plate: SRR14612109 and Mixed: 553 SRR14612108.

Flow-cell biofilms, microscopy and image analysis

556 Biofilms were grown in a flow cell system with continuous flow at a rate of $0.2 \mathrm{~mm} \mathrm{~s}^{-1}$ by a

557 Watson-Marlow 205S peristaltic pump. The flow cell system was assembled as described 558 previously $^{100}$ and liquid $A B$ media supplemented with $0.1 \mathrm{mM}$ sodium citrate was used ${ }^{101}$. 559 Briefly, the flow cell chambers were inoculated with $P$. putida cultures at an $\mathrm{OD}_{600}$ of 0.1 , biofilm 
development was followed every $24 \mathrm{~h}$ up to 5 days. For the competition experiment, the strain

561 inoculated on top of the pre-established biofilm was adjusted to an $\mathrm{OD}_{600}$ of 0.5 . For the two562 species biofilm, strains were mixed in a 1:1 ratio and cultivated for up to $48 \mathrm{~h}$. Shortly before $56340 \mathrm{~h}$ of cultivation, PI was added. Photomicrographs were taken every $24 \mathrm{~h}$ with a confocal 564 laser scanning microscope (CLSM, Leica TCS SPE, DM5500) equipped with a $63 \times 1.3$ oil objective. Single cell competition assays were imaged with a $100 \times 1.44$ oil objective. Images were analysed with the Leica Application Suite, the Imaris 9.6.0 software package (Bitplane) and with Image ${ }^{91}$.

\section{Plant assay}

571 Micro-Tom Solanum lycopersicum L. seeds (Tuinplus bv. Heerenveen, Holland) were surface

572 sterilized with $1 \%$ sodium hypochlorite solution for $10 \mathrm{~min}$, washed 4 times with sterile $\mathrm{dH}_{2} \mathrm{O}$ 573 and placed at $4^{\circ} \mathrm{C}$ for 2 days in the dark. Seeds were then sown on $0.8 \%$ water agar plates and 574 kept at $30^{\circ} \mathrm{C}$ for 2 days in the dark. Germinated seeds were incubated at $22^{\circ} \mathrm{C}$ in long day conditions (16 h light and $8 \mathrm{~h}$ dark, $100 \mathrm{uE}, 60 \% \mathrm{RH}$ ) and seedlings were further grown for 6-7 days until lateral root emergence. Seedlings were injured twice with a $0.4 \mathrm{~mm}$ diameter needle at the root-shoot junction. The roots of the seedlings were then submerged for 10 seconds each

578 in a bacterial suspension set to a final $\mathrm{OD}_{600}$ of 0.5 in $1 \mathrm{mM} \mathrm{MgSO}_{4}$. Inoculated seedlings were 579 next placed on half strength Murashige and Skoog (MS) medium with $1.5 \%$ agar and grown for 58022 days under the long day conditions indicated above. Each tomato plant root was washed 581 and sonicated as previously described ${ }^{102}$ with the following modifications: roots were individually placed in an Eppendorf tube with $750 \mu \mathrm{l}$ of $1 \mathrm{mM} \mathrm{MgSO}_{4}$. The washing step consisted of shaking the tube for $15 \mathrm{~min}$ at $160 \mathrm{rpm}$, followed by sonication for $15 \mathrm{~min}$. The obtained cell suspensions

584 were serial-diluted to allow for CFU quantification. Principal component analysis and 585 hierarchical clustering were performed on individual root weight, shoot area and chlorophyll 586 values. Unit variance scaling was applied and SVD with imputation used to calculate PCs. 587 Prediction ellipses were used to display the 95\% confidence intervals. Root parameters were 
clustered using correlation distance and average linkage, plant samples were clustered using

589 Euclidean distance and average linkage. $n=144$. Three independent biological replicates were

590 performed with a total of 28 plants for each treatment. Shoot area and chlorophyll estimations

591 were obtained from calibrated RGB photographs using Fiji ${ }^{103}$ adapting previous methods ${ }^{104}$.

592 Essentially, individual RGB values were extracted from Blue channel-thresholded plant pictures

593 and normalized to total RGB. Normalized Green and Red channel values were used to calculate

594 a greenness index (Greendex $=4 \mathrm{G}-3 \mathrm{R}$ ). Greendex values and acetone-extracted total

595 chlorophyll per shoot weight measured as previously described ${ }^{105}$ of independent infected or

596 healthy tomato plantlets were linearly correlated $\left(R^{2}=0.7373, n=21\right)$.

597

598

\section{References}

599 1. Syed Ab Rahman, S. F., Singh, E., Pieterse, C. M. J. \& Schenk, P. M. Emerging

600 microbial biocontrol strategies for plant pathogens. Plant Sci. 267, 102-111 (2018).

6012 2. Berendsen, R. L., Pieterse, C. M. J. \& Bakker, P. A. H. M. The rhizosphere

602 microbiome and plant health. Trends Plant Sci. 17, 478-486 (2012).

603 3. Prasad, M., Srinivasan, R., Chaudhary, M., Choudhary, M. \& Jat, L. K. PGPR

$604 \quad$ amelioration in sustainable agriculture Ch. 7. (Elsevier Inc., 2019).

605 4. Raymaekers, K., Ponet, L., Holtappels, D., Berckmans, B. \& Cammue, B. P. A. Screening for novel biocontrol agents applicable in plant disease management - A

608 5. Parnell, J. J. et al. From the lab to the farm: An industrial perspective of plant 609 beneficial microorganisms. Front. Plant Sci. 7, 1-12 (2016).

610 6. Hart, M. M., Antunes, P. M., Chaudhary, V. B. \& Abbott, L. K. Fungal inoculants in the 611 field: Is the reward greater than the risk? Funct. Ecol. 32, 126-135 (2018).

612 7. Mitter, B., Brader, G., Pfaffenbichler, N. \& Sessitsch, A. Next generation microbiome 613 applications for crop production - limitations and the need of knowledge-based

614 solutions. Curr. Opin. Microbiol. 49, 59-65 (2019).

615 8. Busby, P. E. et al. Research priorities for harnessing plant microbiomes in sustainable 
agriculture. PLoS Biol. 15, 1-14 (2017).

617 9. Mitter, E. K., Tosi, M., Obregón, D., Dunfield, K. E. \& Germida, J. J. Rethinking crop nutrition in times of modern microbiology: Innovative biofertilizer technologies. Front. Sustain. Food Syst. 5, 1-23 (2021).

10. Timmusk, S., Behers, L., Muthoni, J., Muraya, A. \& Aronsson, A. C. Perspectives and challenges of microbial application for crop improvement. Front. Plant Sci. 8, 1-10 (2017).

11. Kaminsky, L. M., Trexler, R. V., Malik, R. J., Hockett, K. L. \& Bell, T. H. The inherent conflicts in developing soil microbial inoculants. Trends Biotechnol. 37, 140-151 (2019).

12. Hall-Stoodley, L., Costerton, J. W. \& Stoodley, P. Bacterial biofilms: From the natural environment to infectious diseases. Nat. Rev. Microbiol. 2, 95-108 (2004).

13. Monds, R. D. \& O'Toole, G. A. The developmental model of microbial biofilms: ten years of a paradigm up for review. Trends Microbiol. 17, 73-87 (2009).

14. Compant, S., Clément, C. \& Sessitsch, A. Plant growth-promoting bacteria in the rhizo- and endosphere of plants: Their role, colonization, mechanisms involved and prospects for utilization. Soil Biol. Biochem. 42, 669-678 (2010).

15. Pandin, C., Le Coq, D., Canette, A., Aymerich, S. \& Briandet, R. Should the biofilm mode of life be taken into consideration for microbial biocontrol agents? Microb. Biotechnol. 10, 719-734 (2017).

16. Elias, S. \& Banin, E. Multi-species biofilms: Living with friendly neighbors. FEMS Microbiol. Rev. 36, 990-1004 (2012).

17. Rendueles, O. \& Ghigo, J. M. Multi-species biofilms: How to avoid unfriendly

640 18. Nadell, C. D., Drescher, K., Wingreen, N. S. \& Bassler, B. L. Extracellular matrix structure governs invasion resistance in bacterial biofilms. ISME J. 9, 1700-1709 (2015).

19. Morris, C. E. \& Monier, J. M. The ecological significance of biofilm formation by plant- 
associated bacteria. Annu. Rev. Phytopathol. 41, 429-453 (2003).

645

646

647

648

649

650

651

652

653

654

655

656

657

658

659

660

661

662

663

664

665

666

667

668

669

670

671

20. Danhorn, T. \& Fuqua, C. Biofilm formation by plant-associated bacteria. Annu. Rev. Microbiol. 61, 401-422 (2007).

21. Bakker, P. A. H. M. et al. The soil-borne identity and microbiome-assisted agriculture: Looking back to the future. Mol. Plant 13, 1394-1401 (2020).

22. Benz, J. \& Meinhart, A. Antibacterial effector/immunity systems: It's just the tip of the iceberg. Curr. Opin. Microbiol. 17, 1-10 (2014).

23. Peterson, S. B., Bertolli, S. K. \& Mougous, J. D. The central role of interbacterial antagonism in bacterial life. Curr. Biol. 30, 203-214 (2020).

24. Russell, A. B. et al. Type VI secretion delivers bacteriolytic effectors to target cells. Nature 475, 343-349 (2011).

25. García-Bayona, L., Guo, M. S. \& Laub, M. T. Contact-dependent killing by Caulobacter crescentus via cell surface-associated, glycine zipper proteins. Elife 6, 1-26 (2017).

26. Souza, D. P. et al. Bacterial killing via a type IV secretion system. Nat. Commun. 6, 19 (2015).

27. Bernal, P., Allsopp, L. P., Filloux, A. \& Llamas, M. A. The Pseudomonas putida T6SS is a plant warden against phytopathogens. ISME J. 11, 972-987 (2017).

28. Ahmad, S. et al. An interbacterial toxin inhibits target cell growth by synthesizing (p)ppApp. Nature 575, 674-678 (2019).

29. Whitney, J. C. et al. An interbacterial $\operatorname{NAD}(P)+$ glycohydrolase toxin requires elongation factor Tu for delivery to target cells. Cell 163, 607-619 (2015).

30. Ting, S. Y. et al. Bifunctional immunity proteins protect bacteria against FtsZ-targeting ADP-ribosylating toxins. Cell 175, 1380-1392 (2018).

31. Klein, T. A., Ahmad, S. \& Whitney, J. C. Contact-dependent interbacterial antagonism mediated by protein secretion machines. Trends Microbiol. 28, 387-400 (2020).

32. Yang, X., Long, M. \& Shen, X. Effector-immunity pairs provide the T6SS nanomachine its offensive and defensive capabilities. Molecules 23, (2018).

33. Granato, E. T., Meiller-Legrand, T. A. \& Foster, K. R. The evolution and ecology of 
bacterial warfare. Curr. Biol. 29, R521-R537 (2019).

673

674

675

676

677

678

679

680

681

682

683

684

685

686

687

688

689

690

691

692

693

694

695

696

697

698

699

34. Willett, J. L. E., Ruhe, Z. C., Goulding, C. W., Low, D. A. \& Hayes, C. S. Contactdependent growth inhibition (CDI) and $\mathrm{CdiB} / \mathrm{CdiA}$ two-partner secretion proteins. J. Mol. Biol. 427, 3754-3765 (2015).

35. Ruhe, Z. C., Low, D. A. \& Hayes, C. S. Bacterial contact-dependent growth inhibition. Trends Microbiol. 21, 230-237 (2013).

36. Ho, B. T., Dong, T. G. \& Mekalanos, J. J. A view to a kill: The bacterial type VI secretion system. Cell Host Microbe 15, 9-21 (2014).

37. Cianfanelli, F. R., Monlezun, L. \& Coulthurst, S. J. Aim, load, fire: The type VI secretion system, a bacterial nanoweapon. Trends Microbiol. 24, 51-62 (2016).

38. Basler, M., Pilhofer, M., Henderson, G. P., Jensen, G. J. \& Mekalanos, J. J. Type VI secretion requires a dynamic contractile phage tail-like structure. Nature $483,182-186$ (2012).

39. Backert, S., Grohmann, E. \& (eds). Type IV Secretion in Gram-Negative and GramPositive Bacteria, Current Topics in Microbiology and Immunology. (Springer, 2017).

40. Berger, K. H. \& Isberg, R. R. Two distinct defects in intracellular growth complemented by a single genetic locus in Legionella pneumophila. Mol. Microbiol. 7, 7-19 (1993).

41. Brand, B. C., Sadosky, A. B. \& Shuman, H. A. The Legionella pneumophila icm locus: a set of genes required for intracellular multiplication in human macrophages. Mol. Microbiol. 14, 797-808 (1994).

42. Voth, D. E., Broederdorf, L. J. \& Graham, J. Bacterial type IV secretion systems: Versatile virulence machines. Future Microbiol. 7, 241-257 (2012).

43. Christie, P. J. \& Vogel, J. P. Bacterial type IV secretion: Conjugation systems adapted to deliver effector molecules to host cells. Trends Microbiol. 8, 354-360 (2000).

44. Hubber, A. \& Roy, C. R. Modulation of host cell function by Legionella pneumophila type IV effectors. Annu. Rev. Cell Dev. Biol. 26, 261-283 (2010).

45. Steidle, A. et al. Identification and characterization of an N-acylhomoserine lactonedependent quorum-sensing system in Pseudomonas putida strain IsoF. Appl. Environ. 
Microbiol. 68, 6371-6382 (2002).

701

702

703

704

705

706

707

708

709

710

711

712

713

714

715

716

717

718

719

720

721

722

723

46. Steidle, A. et al. Visualization of N-acylhomoserine lactone-mediated cell-cell communication between bacteria colonizing the tomato rhizosphere. Appl. Environ. Microbiol. 67, 5761-5770 (2001).

47. Espinosa-Urgel, M., Salido, A. \& Ramos, J. L. Genetic analysis of functions involved in adhesion of Pseudomonas putida to seeds. J. Bacteriol. 182, 2363-2369 (2000).

48. Kubori, T. \& Nagai, H. The type IVB secretion system: An enigmatic chimera. Curr. Opin. Microbiol. 29, 22-29 (2016).

49. Nagai, H. \& Kubori, T. Type IVB secretion systems of Legionella and other Gramnegative bacteria. Front. Microbiol. 2, 1-12 (2011).

50. Zatyka, M. \& Thomas, C. M. Control of genes for conjugative transfer of plasmids and other mobile elements. FEMS Microbiol. Rev. 21, 291-319 (1998).

51. Vincent, C. D. et al. Identification of the core transmembrane complex of the Legionella Dot/lcm type IV secretion system. Mol. Microbiol. 62, 1278-1291 (2006).

52. Nakano, N., Kubori, T., Kinoshita, M., Imada, K. \& Nagai, H. Crystal structure of Legionella DotD: Insights into the relationship between type IVB and type II/III secretion systems. PLoS Pathog. 6, e1001129 (2010).

53. Yerushalmi, G., Zusman, T. \& Segal, G. Additive effect on intracellular growth by Legionella pneumophila Icm/Dot proteins containing a lipobox motif. Infect. Immun. 73, 7578-7587 (2005).

54. Liu, M. et al. ICEberg 2.0: An updated database of bacterial integrative and conjugative elements. Nucleic Acids Res. 47, D660-D665 (2019).

55. Hood, R. D. et al. A Type VI secretion system of Pseudomonas aeruginosa targets a toxin to bacteria. Cell Host Microbe 7, 25-37 (2010).

56. Pissaridou, P. et al. The Pseudomonas aeruginosa T6SS-VgrG1b spike is topped by a PAAR protein eliciting DNA damage to bacterial competitors. Proc. Natl. Acad. Sci. U. S. A. 115, 12519-12524 (2018).

57. Liang, X. et al. An onboard checking mechanism ensures effector delivery of the type 
VI secretion system in Vibrio cholerae. Proc. Natl. Acad. Sci. U. S. A. 116, 2329223298 (2019).

58. Dong, T. G., Ho, B. T., Yoder-Himes, D. R. \& Mekalanos, J. J. Identification of T6SSdependent effector and immunity proteins by Tn-seq in Vibrio cholerae. Proc. Natl. Acad. Sci. U. S. A. 110, 2623-2628 (2013).

59. Nolan, L. M. et al. Identification of Tse8 as a Type VI secretion system toxin from Pseudomonas aeruginosa that targets the bacterial transamidosome to inhibit protein

60. Solaimanpour, S., Sarmiento, F. \& Mrázek, J. Tn-seq explorer: A tool for analysis of high-throughput sequencing data of transposon mutant libraries. PLoS One 10, 1-15 (2015).

61. Xue, H., Lozano-Durán, R. \& Macho, A. P. Insights into the root invasion by the plant

62. Lowe-Power, T. M., Khokhani, D. \& Allen, C. How Ralstonia solanacearum exploits and thrives in the flowing plant xylem environment. Trends Microbiol. 26, 929-942 (2018).

63. Bayer-Santos, E. et al. The opportunistic pathogen Stenotrophomonas maltophilia utilizes a type IV secretion system for interbacterial killing. PLoS Pathog. 15, 1-29 (2019).

64. Harms, A. et al. A bacterial toxin-antitoxin module is the origin of inter-bacterial and inter-kingdom effectors of Bartonella. PLoS Genet. 13, 1-22 (2017).

65. Sgro, G. G. et al. Bacteria-killing type IV secretion systems. Front. Microbiol. 10, 1-20 (2019).

66. Qin, T., Zhou, H., Ren, H. \& Liu, W. Distribution of secretion systems in the genus Legionella and its correlation with pathogenicity. Front. Microbiol. 8, 1-12 (2017).

67. Basler, M. \& Mekalanos, J. J. Type 6 secretion dynamics within and between bacterial cells. Science 337,815 (2012). 
secretion system-mediated immunity to type 4 secretion system-mediated gene transfer. Science 342, 250-253 (2013).

69. Basler, M., Ho, B. T. \& Mekalanos, J. J. Tit-for-tat: Type VI secretion system counterattack during bacterial cell-cell interactions. Cell 152, 884-894 (2013).

70. Stolle, A. S., Meader, B. T., Toska, J. \& Mekalanos, J. J. Endogenous membrane stress induces T6SS activity in Pseudomonas aeruginosa. Proc. Natl. Acad. Sci. U. S. A. 118, 1-12 (2021).

71. Spiewak, H. L. et al. Burkholderia cenocepacia utilizes a type VI secretion system for bacterial competition. Microbiologyopen 8, e00774 (2019).

72. Chien, C. F. et al. HSI-II gene cluster of Pseudomonas syringae pv. tomato DC3000 encodes a functional type VI secretion system required for interbacterial competition. Front. Microbiol. 11, 1118 (2020).

73. Molina-Santiago, C. et al. The extracellular matrix protects Bacillus subtilis colonies from Pseudomonas invasion and modulates plant co-colonization. Nat. Commun. 10,

74. Durán, D. et al. Pseudomonas fluorescens F113 type VI secretion systems mediate bacterial killing and adaption to the rhizosphere microbiome. Sci. Rep. 11, 1-13 (2021).

75. Shyntum,

, D. Y. et al. The impact of type VI secretion system, bacteriocins and antibiotics on bacterial competition of Pectobacterium carotovorum subsp. brasiliense and the regulation of carbapenem biosynthesis by iron and the ferric-uptake regulator. Front. Microbiol. 10, 2379 (2019).

76. Tian, Y. et al. Type VI secretion systems of Erwinia amylovora contribute to bacterial competition, virulence, and exopolysaccharide production. Phytopathology 107, 654661 (2017).

781 77. Schwarz, S. et al. Burkholderia type VI secretion systems have distinct roles in eukaryotic and bacterial cell interactions. PLoS Pathog. 6, 77-78 (2010).

783 78. Nadell, C. D., Drescher, K. \& Foster, K. R. Spatial structure, cooperation and 
competition in biofilms. Nat. Rev. Microbiol. 14, 589-600 (2016).

79. Hibbing, M. E., Fuqua, C., Parsek, M. R. \& Peterson, S. B. Bacterial competition: Surviving and thriving in the microbial jungle. Nat. Rev. Microbiol. 8, 15-25 (2010).

80. Pandit, A., Adholeya, A., Cahill, D., Brau, L. \& Kochar, M. Microbial biofilms in nature: unlocking their potential for agricultural applications. J. Appl. Microbiol. 129, 199-211 (2020).

81. Krishna Kumar, R. et al. Droplet printing reveals the importance of micron-scale structure for bacterial ecology. Nat. Commun. 12, 1-12 (2021).

82. Kuiper, I. et al. Characterization of two Pseudomonas putida lipopeptide biosurfactants, putisolvin I and II, which inhibit biofilm formation and break down existing biofilms. Mol. Microbiol. 51, 97-113 (2004).

83. Gotschlich, A. et al. Synthesis of multiple N-acylhomoserine lactones is wide-spread among the members of the Burkholderia cepacia complex. Syst. Appl. Microbiol. 24, $1-14(2001)$

84. Zhang, R., Vivanco, J. M. \& Shen, Q. The unseen rhizosphere root-soil-microbe interactions for crop production. Curr. Opin. Microbiol. 37, 8-14 (2017).

85. Clark, D. J. \& Maaløe, O. DNA replication and the division cycle in Escherichia coli. J. Mol. Biol. 23, 99-112 (1967).

86. Lambertsen, L., Sternberg, C. \& Molin, S. Mini-Tn7 transposons for site-specific tagging of bacteria with fluorescent proteins. Environ. Microbiol. 6, 726-732 (2004).

87. Choi, K.-H. \& Schweizer, H. P. Mini-Tn7 insertion in bacteria with single attTn7 sites: example Pseudomonas aeruginosa. Nat. Protoc. 1, 153-161 (2006).

88. Aguilar, C., Schmid, N., Lardi, M., Pessi, G. \& Eberl, L. The IcIR-family regulator BapR controls biofilm formation in B. cenocepacia H111. PLoS One 9, 1-7 (2014).

89. Huber, B. et al. Genetic analysis of functions involved in the late stages of biofilm development in Burkholderia cepacia H111. Mol. Microbiol. 46, 411-426 (2002).

90. de Lorenzo, V. \& Timmis, K. N. Analysis and construction of stable phenotypes in gram-negative bacteria with Tn5- and Tn10-derived minitransposons. Methods 
Enzymol. 235, 386-405 (1994).

813 91. Schneider, C. A., Rasband, W. S. \& Eliceiri, K. W. NIH Image to ImageJ: 25 years of 814 image analysis. Nat. Methods 9, 671-675 (2012).

815 92. Flannagan, R. S., Linn, T. \& Valvano, M. A. A system for the construction of targeted unmarked gene deletions in the genus Burkholderia. Environ. Microbiol. 10, 16521660 (2008).

93. Darling, A. C. E., Mau, B., Blattner, F. R. \& Perna, N. T. Mauve: Multiple alignment of conserved genomic sequence with rearrangements. Genome Res. 14, 1394-1403

94. Medema, M. H., Takano, E. \& Breitling, R. Detecting sequence homology at the gene cluster level with MultiGeneBlast. Mol. Biol. Evol. 30, 1218-1223 (2013).

95. Gallagher, L. A., Shendure, J. \& Manoil, C. Genome-scale identification of resistance functions in Pseudomonas aeruginosa using Tn-seq. MBio 2, 1-8 (2011).

96. Higgins, S., Gualdi, S., Pinto-Carbó, M. \& Eberl, L. Copper resistance genes of Burkholderia cenocepacia $\mathrm{H} 111$ identified by transposon sequencing. Environ. Microbiol. Rep. 12, 241-249 (2020).

97. Bolger, A. M., Lohse, M. \& Usadel, B. Trimmomatic: A flexible trimmer for Illumina sequence data. Bioinformatics 30, 2114-2120 (2014).

98. Martin, M. Cutadapt removes adapter sequences from high-throught sequencing reads. EMBnet.journal 17, (2011).

99. Langmead, B. \& Salzberg, S. L. Fast gapped-read alignment with Bowtie 2. Nat. Methods 9, 357-359 (2012).

834 100. Christensen, B. B. et al. Molecular tools for study of biofilm physiology. Methods Enzymol. 310, 20-42 (1999).

836 101. Heydorn, A. et al. Experimental reproducibility in flow-chamber biofilms. Microbiology 146, 2409-2415 (2000).

838 102. Bulgarelli, D. et al. Revealing structure and assembly cues for Arabidopsis rootinhabiting bacterial microbiota. Nature 488, 91-95 (2012). 
103. Schindelin, J. et al. Fiji: An open-source platform for biological-image analysis. Nat. Methods 9, 676-682 (2012).

104. Liang, Y. et al. A nondestructive method to estimate the chlorophyll content of Arabidopsis seedlings. Plant Methods 13, 1-10 (2017).

105. Lichtenthaler, H. K. \& Wellburn, A. R. Determinations of total carotenoids and chlorophylls a and b of leaf extracts in different solvents. Biochem. Soc. Trans. 11, 591-592 (1983).

106. Chetrit, D., Hu, B., Christie, P. J., Roy, C. R. \& Liu, J. A unique cytoplasmic ATPase complex defines the Legionella pneumophila type IV secretion channel. Nat. Microbiol. 3, 678-686 (2018).

\section{Acknowledgments}

This work was funded in part by the Swiss National Science Foundation grants CRSII5_186410 and 310030_192800. We would like to thank Claudio Aguilar for his assistance in constructing the IsoF mini-Tn5 library and Alessandra Vitale for help with the TnSeq. We would like to thank Carlotta Fabbri, Joel Steger and Elina Leu for technical support. We are grateful to Yi-chi Chen and Stefano Gualdi for helpful discussions and Kirsty Agnoli for improving the English.

\section{Author contributions}

GP-M, GC-O and LE designed the overall experimental plan for the manuscript. GP-M performed the majority of the experiments presented and wrote the manuscript with input from all authors. GC-O contributed to project management and performed the Tn5 library experiments, the initial bacterial competition experiments and the flow cell biofilm experiments with the wild type strains. MP-C contributed to the analysis of the TnSeq library, bioinformatic analyses, and the sequencing and annotation of the genome of IsoF. KA supported all molecular microbiology experiments and contributed to writing the manuscript. AB contributed to the design of the plant experiment and performed the principal component analysis, 
867 hierarchical clustering and related analyses. LE contributed to project management and to 868 writing the manuscript. Competing interests: The authors declare no competing interests. 
a
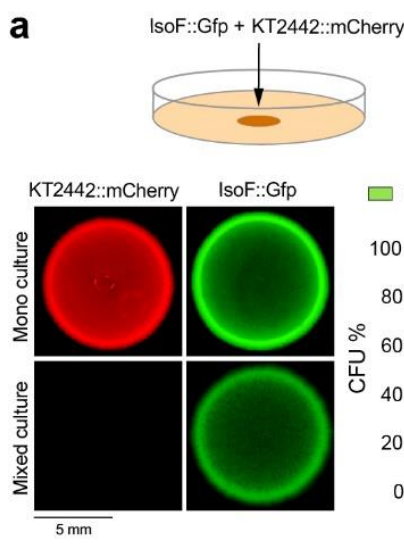

d

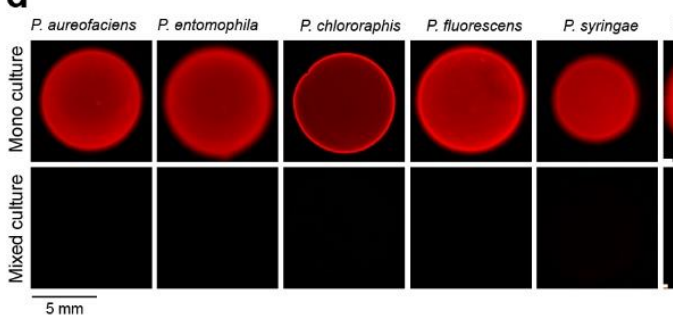

b
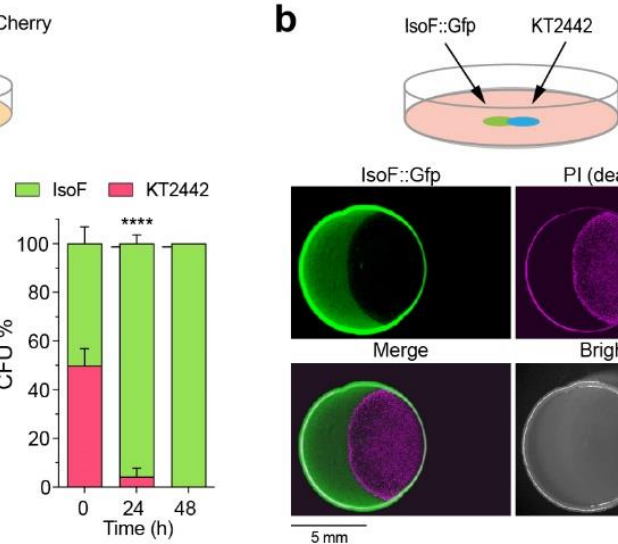

Merge

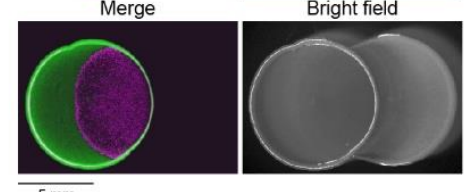

e
C

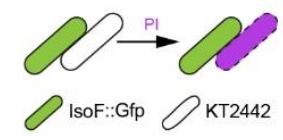

PI (dead cells)

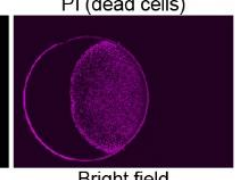

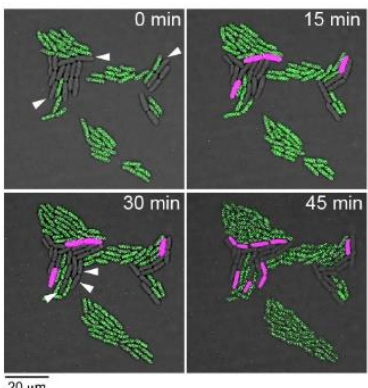

- IsoF - Competitor
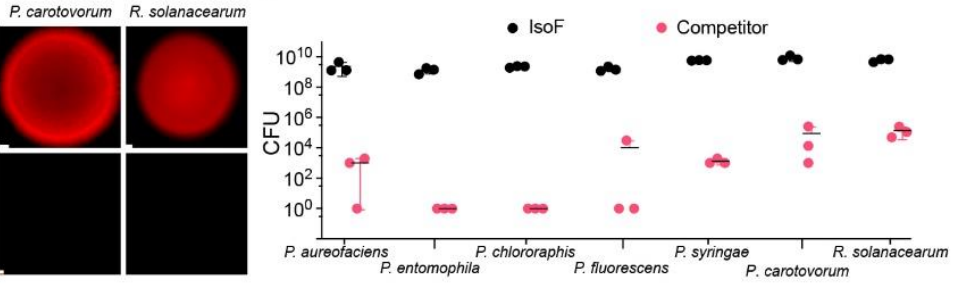

Figure 1. IsoF displays contact-dependent antagonism against a wide range of Gram-

negative bacteria a, IsoF::Gfp outcompetes KT2442::mCherry after co-inoculation on ABC agar

872 plates. Fluorescence is indicative of viable bacteria. The percentage of the CFUs of each bacterial

873 population in the mixed culture at $0 \mathrm{~h}, 24 \mathrm{~h}$, and $48 \mathrm{~h}$ is shown. Data are mean \pm s.d. from 3

874 biological replicates $(n=3)$. Unpaired t-test, ${ }^{\star \star \star \star} P<0.0001$. b, IsoF antagonism is restricted to

875 areas where IsoF::Gfp and KT2442 colonies are in direct contact. The medium was supplemented

876 with propidium iodide (PI) to visualize dead cells. c, IsoF::Gfp kills KT2442 cells in a contact-

877 dependent manner. Cell death was monitored by PI staining (cells shown in magenta). d, IsoF

878 kills a wide range of Gram-negative plant-associated bacteria, including $P$. aureofaciens, $P$.

879 entomophila, $P$. chlororaphis, $P$. fluorescens, $P$. syringae, $P$. carotovorum, and $R$. solanacearum.

880 All competitors were tagged with mCherry. e, CFUs were determined after $24 \mathrm{~h}$ of competition.

881 Data are mean \pm s.d. from 3 independent biological replicates $(n=3)$. 
a

P. aureofaciens: mCherry 0 soF

- 2000 mini-Tn5-insertion mutants

$P$. aureofaciens: $m$ Cherry in mixed cultures

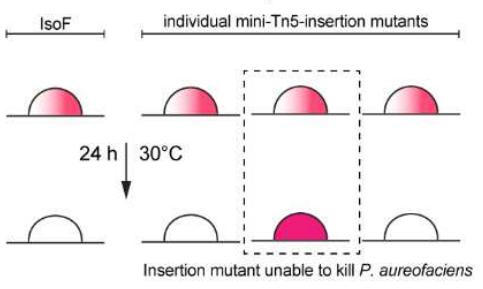

C

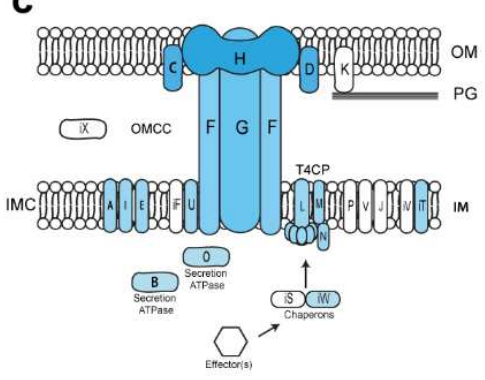

b
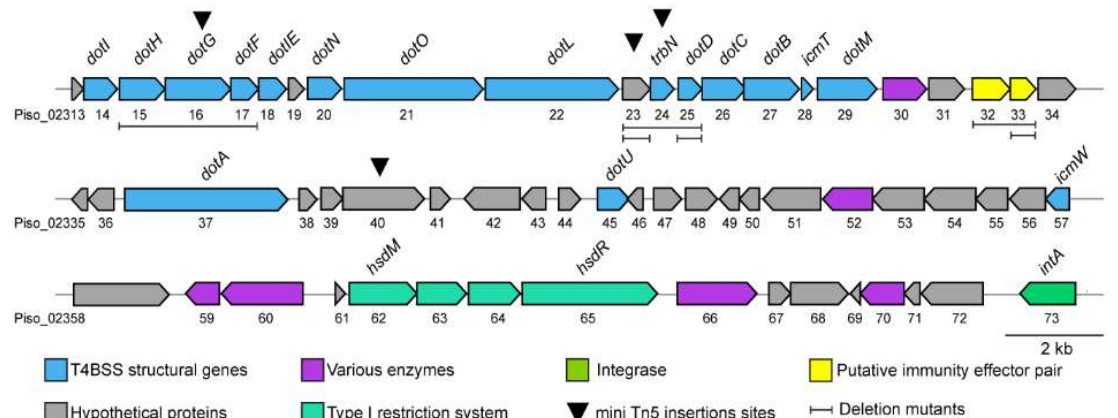

d

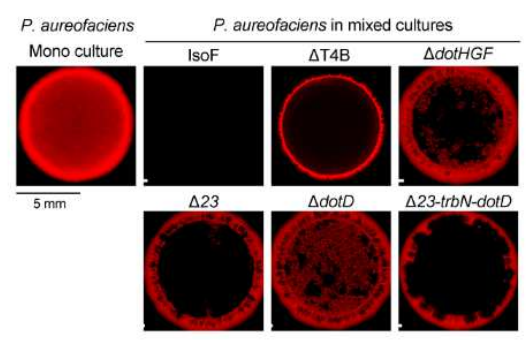

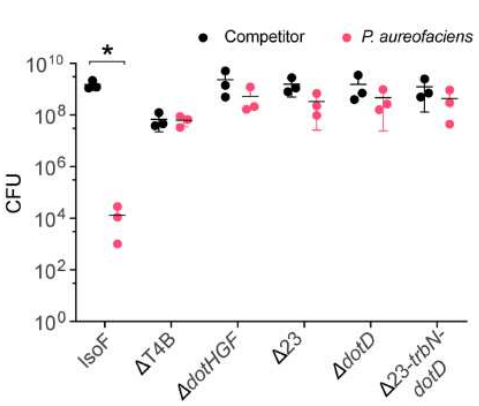

883

884

Figure 2. The IsoF T4BSS-dependent killing machine is encoded on a genomic island (GI).

a, Single IsoF-Tn5-insertion mutants were mixed with $P$. aureofaciens tagged with mCherry. Red fluorescence indicates the loss of killing activity by the mutant. $\mathbf{b}$, Genetic organization of the IsoF GI that encodes a T4BSS required for bacterial killing. The cluster has a length of $69.9 \mathrm{~kb}$ and codes for 17 T4BSS structural and 34 hypothetical proteins. Additionally, the 3' end encodes a Type I RM system and an integrase. Regions that were deleted in defined mutants are underlined in black: $\triangle$ dotHGF, $\Delta 23, \Delta \operatorname{dot} D, \Delta 23-\operatorname{trbN-dotD}, \Delta 33$, and $\Delta 32-33$. c, Architecture of the T4BSS gene cluster in Legionella. Homologs of proteins highlighted in blue are encoded by the IsoF-GI. In the Legionella Icm/Dot system, effector molecules bind to chaperons which interact with the type 4 coupling protein (T4CP) before the effector molecules are translocated through the outer membrane core complex. The two ATPases provide energy and interact with the inner membrane complex (IMC) at the substrate recognition and translocation domains (modified from ${ }^{39,106}$ ). d, Fluorescence images show $P$. aureofaciens::mCherry in competition with the IsoF wild type and various deletion mutants after $24 \mathrm{~h}$ of co-incubation. CFUs of the two competing strains. Data are mean \pm s.d. of three independent biological replicates $(n=3)$. Unpaired t-test, ${ }^{*} P<0.05$. 
a

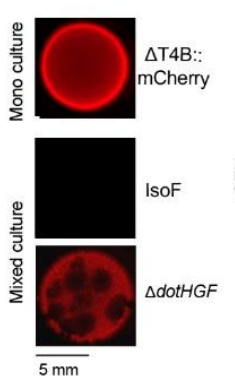

d

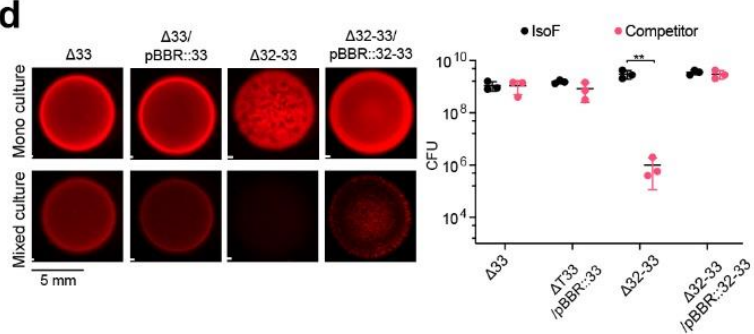

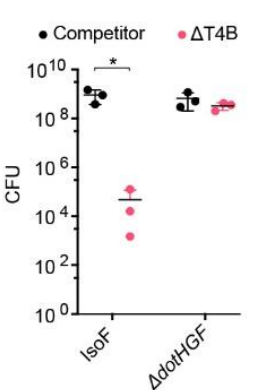

b IsoF

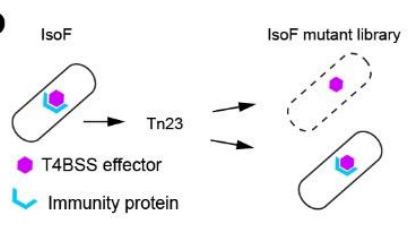

C

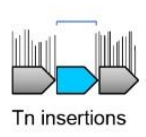

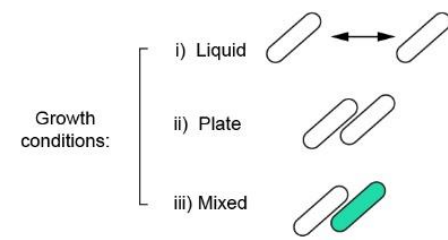

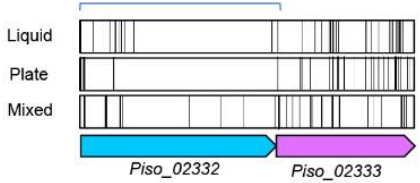

e
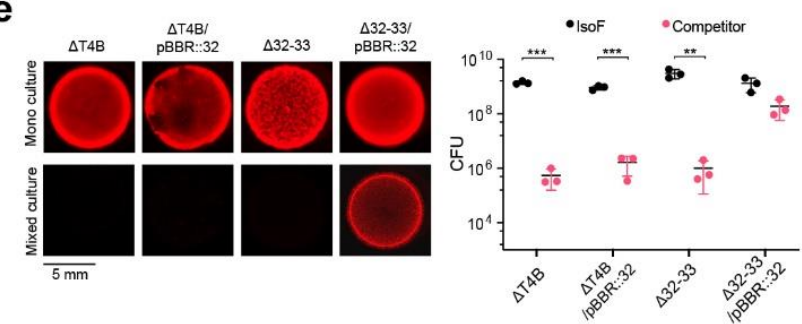

899

900

901

902

903

904

905

906

907

908

909

910

911

912

913

914

915

Figure 3. An E-I pair is encoded within the kib gene cluster. a, Contact-dependent competition of the $\Delta \mathrm{T} 4 \mathrm{~B}$ mutant against the IsoF wild type and the $\Delta$ dotHGF deletion mutant. Representative fluorescence images of three independent experiments are shown. $\mathbf{b}$, An effector will be toxic for the cell in the absence of its cognate immunity protein. The following conditions were used to challenge an IsoF Tn23 mutant library: (i) growth in liquid medium with shaking to prevent cell-tocell contact, (ii) growth on an agar surface either alone or (iii) in the presence of the competitor $P$. aureofaciens to promote competition (mixed). c, The unique insertion density approach of the TnSeq explorer software was used to identify genes that provide a fitness benefit for growth under the applied growth conditions ${ }^{60}$. Piso_02332 (blue) was found to have very few transposon insertions under all three culture conditions. A putative effector gene, Piso_02333 (magenta), is located downstream of Piso_02332. The Tn23 insertions in the two genes for the three growth conditions are shown. $\mathbf{d}, \mathrm{CDC}$ of IsoF against the deletion mutants $\Delta 32-33$ (lacking the $\mathrm{E}$-I pair). $\Delta 33$ (lacking the effector gene) and their complemented derivatives. e, CDC of the IsoF wild type against mutants $\Delta T 4 B / p B B R:: 32$ and $\Delta 32-33 / p B B R:: 32$. Representative fluorescence images of three independent experiments are shown. CFUs of the competing strains were determined after $24 \mathrm{~h}$ incubation. Data are mean \pm s.d. of three independent replicates $(\mathrm{n}=3)$. Unpaired t-test, ${ }^{*} P<$ $0.05 ;{ }^{* *} P<0.01 ;{ }^{* *} P<0.001$. 
a
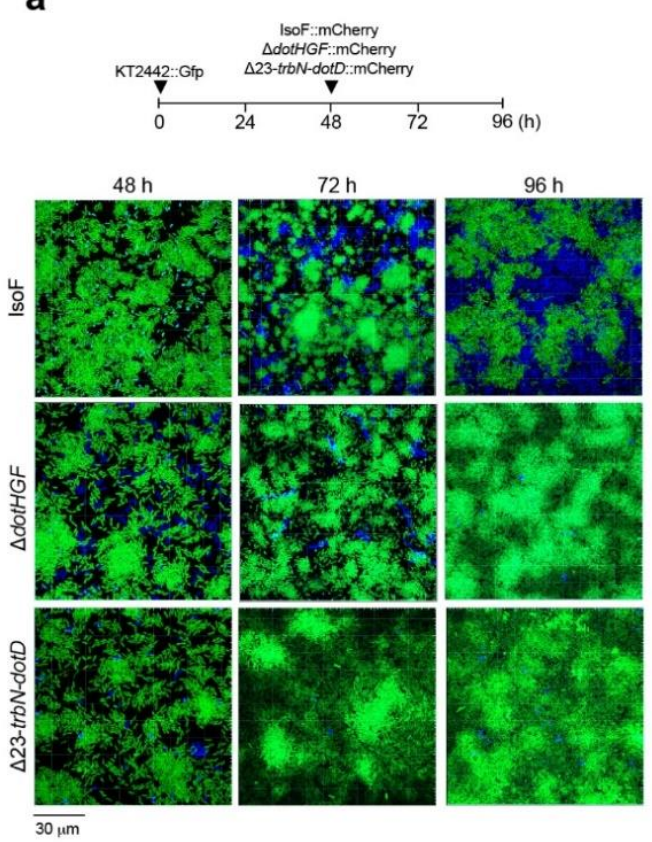

b

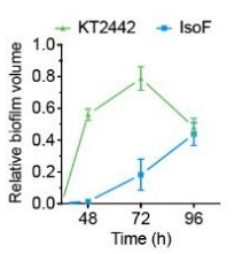

C
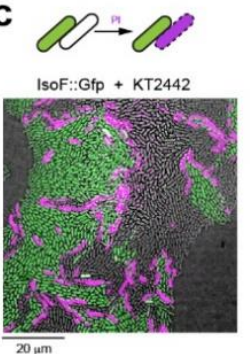

e $\quad 22 \mathrm{~h}$

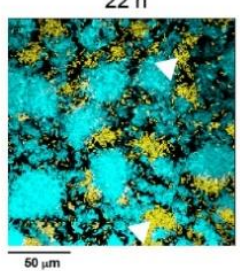

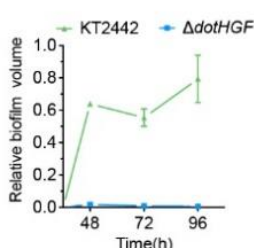

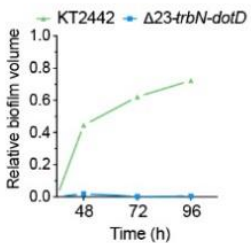

d

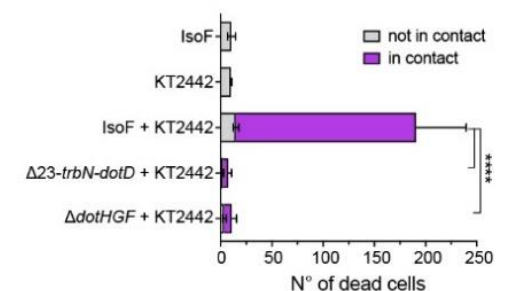

f
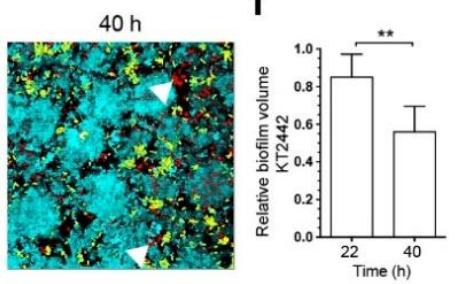

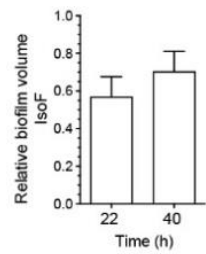

917

Figure 4. IsoF wild type invades and displaces a pre-established KT2442 biofilm. a, A two day-old biofilm of KT2442::Gfp (green) is invaded by IsoF::mCherry (blue) but not by $\Delta$ dotHGF::mCherry (blue) or $\Delta 23$-trbN-dotD::mCherry (blue). b, Relative biofilm biomass (volume) of KT2442::Gfp relative to IsoF::mCherry, $\triangle$ dotHGF::mCherry and $\Delta 23-t r b N$ dotD::mCherry. Data are mean \pm s.d. from up to three biological replicates $(n=3)$. c, Competition of IsoF::Gfp against KT2442 after $18 \mathrm{~h}$ of incubation. Dead cells (magenta) were visualized by staining with PI. d, The number of dead cells in contact and not in contact with a green fluorescent cell were quantified from at least 9 randomly chosen images from the different competition experiments. As a control, the strains were also inoculated without a competitor and the number of dead cells was determined. Data are mean \pm s.d. of three independent replicates $(n=3)$. Unpaired t-test, ${ }^{* * *} P<0.0001$. e, Mixed biofilm of KT2442::Gfp (yellow) and IsoF::Cfp (cyan) after $40 \mathrm{~h}$ of co-cultivation. Dead cells (red) were visualized by staining with PI. f, Relative biofilm volumes of KT2442 and IsoF at 22 and $40 \mathrm{~h}$. Unpaired t-test, ${ }^{* *} P<0.01$. 
a

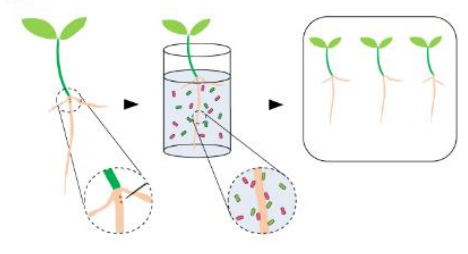

d

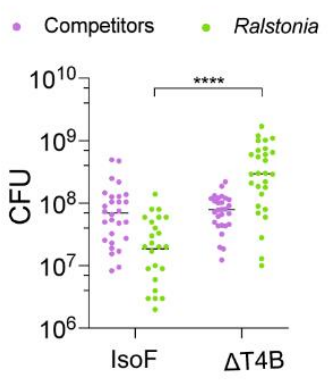

b

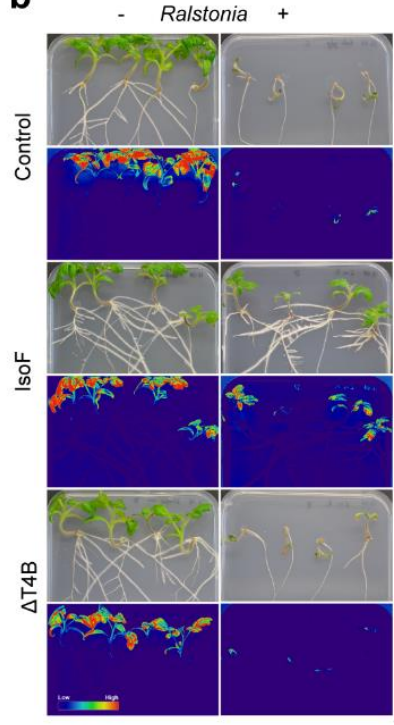

C

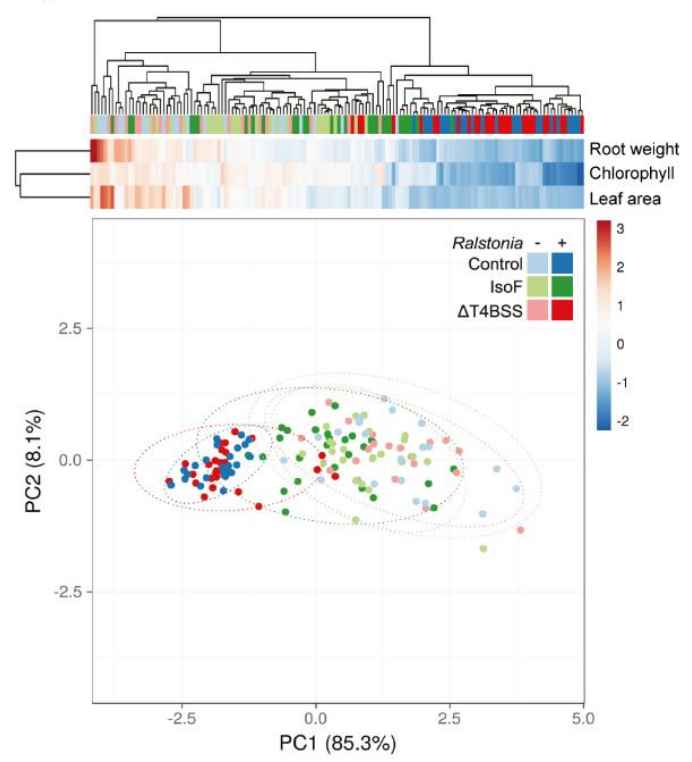

932 Figure 5. IsoF-T4BSS protects tomato plants against bacterial wilt. a, Schematic

933 representation of the experimental approach used. Seedlings were injured twice with a needle in

934 the root-shoot junction and immersed in the bacterial suspensions. Inoculated seedlings were

935 transferred to $1 / 2$ MS plates for further growth. $\mathbf{b}$, Representative images of tomato plants 22 days

936 after inoculation. False color pictures display the green component of the RGB pictures to

937 estimate chlorophyll content. c, Principal component analysis and hierarchical clustering heatmap

938 of estimated plant health parameters. d, CFUs of recovered bacterial competitor cells from the

939 tomato roots. In total 28 plants were assessed with a minimum of 8 plants per treatment for each

940 of the three independent replicates $(\mathrm{n}=3)$. Unpaired t-test, ${ }^{* * * *} P<0.0001$. 


\section{Supplementary Files}

This is a list of supplementary files associated with this preprint. Click to download.

- Supplementarylnformation.pdf

- ExtendedData.pdf

- ExtendedDataVideo1medium.mp4 